\title{
Le paradis, le mariage et la terre
}

Des langues de l'écrit en milieu fuutanke (arabe, français et pulaar)

Paradise, Marriage and Earth: Literacy, Writings and Languages in Fuutanke Area (Arabic, French and Fula)

\section{Marie-Ève Humery-Dieng}

\section{OpenEdition \\ Journals}

Édition électronique

URL : http://journals.openedition.org/etudesafricaines/110

DOI : 10.4000/etudesafricaines. 110

ISSN : $1777-5353$

Éditeur

Éditions de l'EHESS

\section{Édition imprimée}

Date de publication : 1 janvier 2001

Pagination : 565-594

ISBN : 978-2-7132-1394-6

ISSN : 0008-0055

Référence électronique

Marie-Ėve Humery-Dieng, "Le paradis, le mariage et la terre », Cahiers d'études africaines [En ligne], 163-164 | 2001, mis en ligne le 21 novembre 2013, consulté le 05 février 2021. URL : http://

journals.openedition.org/etudesafricaines/110; DOI : https://doi.org/10.4000/etudesafricaines.110 


\section{Marie-Ève Humery-Dieng}

\section{Le paradis, le mariage et la terre : des langues de l'écrit en milieu fuutanke (arabe, français et pulaar)}

Depuis plus ou moins deux décennies, l'une des principales langues africaines en termes de nombre de locuteurs ${ }^{1}$ et d'aire géographique - le pulaar ou fulfulde (langue peule) - connaît un phénomène socioculturel assez remarquable de par son ampleur, et ce dans plusieurs des zones de la bande soudano-sahélienne où elle est parlée. Dans l'une d'elles, le Fuuta Tooro, ce phénomène prit la forme de véritables résistances identitaire et culturelle qui puisèrent leur source dans le militantisme d'intellectuels, de fonctionnaires et de groupements de jeunes qui se retrouvèrent à Dakar, Saint-Louis ou Nouakchott. Ils étaient quasiment tous originaires de cette région du Fuuta partagée depuis les indépendances entre les territoires mauritaniens et sénégalais et correspondant à la Moyenne Vallée du fleuve Sénégal. Ce «mouvement pulaar» (Humery 1997) s'ancra localement sur deux axes d'activités simultanées : la sensibilisation des villageois fuutanke sur l'importance de leur langue maternelle face à l'arabisation forcée d'une part (en Mauritanie) et à la wolofisation relativement spontanée d'autre part (au Sénégal) et l'alphabétisation massive — toutes proportions gardées — des Haalpulaar'en dans leur langue.

Or, si le pulaar n'a pas attendu le colonisateur français pour se scripturaliser, puisqu'il utilisait des graphies arabes dès le XIX ${ }^{\mathrm{e}}$ siècle, il s'est bel et bien choisi, pour se populariser sous sa forme écrite actuelle, notamment à travers ce «mouvement », une graphie latine adaptée à sa phonologie ${ }^{2}$. Par qui et pourquoi cette orientation vers les caractères latins s'est-elle opérée, et avec quelles conséquences socioculturelles ? Ajoutez à cela deux aspects

1. 20 à 30 millions de locuteurs selon les estimations, répartis sur près d'une vingtaine de pays du continent, quasiment de l'océan Atlantique jusqu'au Nil.

2. L'alphabet utilisé est inspiré de la conférence tenue sous l'égide de l'UNESCO à Bamako en 1966 qui se fixait pour mission d'accorder les experts en linguistiques ouest-africaines sur des graphies communes, standardisables et adaptées aux principales langues de la sous-région. 
de l'histoire de la présence du mode écrit dans la région et l'on comprendra combien il devient palpitant tout autant que complexe et «politiquement » sensible d'appréhender les relations entre les trois langues de l'écrit de la zone - arabe, français et pulaar. Le premier de ces aspects est que le Fuuta Tooro s'est trouvé être parmi les premières zones de contact de l'Afrique subsaharienne avec l'islam dès le $\mathrm{x}^{\mathrm{e}}$ siècle ${ }^{3}$, et donc très tôt en contact avec des écrits en arabe et elle-même productrice d'une littérature religieuse et de documents diplomatiques dans la langue du Prophète. Certes, pendant longtemps l'arabe et ses écrits étaient le strict fait d'une élite aristocratique religieuse et politique au sein de cette société haalpulaar structurée en groupes statutaires ou «castes » et ce n'est qu'au cours du XIX ${ }^{\mathrm{e}}$ siècle que l'islam devint la religion de l'ensemble des Haalpulaar'en. Mais, aujourd'hui, le Fuuta est considéré comme le berceau de l'islam subsaharien et l'un de ses fiefs, et les Fuutankoobe ${ }^{4}$ véhiculent l'image d'une grande piété dans des contextes de populations nationales déjà presque entièrement islamisées. Le second aspect est que neuf siècles plus tard, la colonisation française y implanta, non sans résistance, la langue de Molière (à quelques variantes près ? !) qui fut conservée après les Indépendances comme langue officielle et langue de l'Éducation nationale de façon constante sur la rive sénégalaise et discontinue sur la rive mauritanienne.

Comment dans ce contexte historique de double pénétration scripturolinguistique ${ }^{5}$ peut s'opérer l'avènement à l'écrit de la langue maternelle ? Quelles peuvent être les incidences directes ou indirectes de cet avènement pour la société concernée et les rapports de cette langue maternelle avec les autres langues et cultures en contact ? Comment les individus aussi bien que les différents acteurs et groupes sociaux disent-ils et gèrent-ils leurs rapports d'implication ou de non-implication vis-à-vis des filières éducatives chargées de transmettre les compétences scripturales et/ou linguistiques évoquées ( «écoles coraniques » ou dude, école publique et cours d'alphabétisation) ? Et comment et pourquoi ces derniers choisissent-ils — si libre choix il y a - de développer telle ou telle langue de/à l'écrit ? C'est donc tout le jeu de relations et d'interactions entre systèmes de langues écrites et

3. Les différentes sources historiques font remonter les débuts de l'islamisation du Tekrur, via le commerce transsaharien, aux $\mathrm{IX}^{\mathrm{e}}, \mathrm{x}^{\mathrm{e}}$ ou XI $\mathrm{xI}^{\mathrm{e}}$ siècles. Selon M'BoKolo (1992: 105), cette région aurait été islamisée au $\mathrm{X}^{\mathrm{e}}$ siècle, après Gao et les pays tchadiens.

4. Habitants du Fuuta, littéralement «ceux du Fuuta ».

5. Étant donné notre sujet d'étude, nous ne pouvons bien souvent pas dissocier la dimension linguistique au sens de connaissance d'une langue - à l'oral et à l'écrit - de la dimension spécifiquement scripturale. Nous serons donc amenée à utiliser ces adjectifs un peu barbares de «scripturo-linguistique » ou de «linguistico-scriptural » qui ont des sens légèrement distincts : le premier met l'accent sur la dimension linguistique, mais pour les langues de l'écrit, et le second insiste au contraire sur la dimension écrite ou liée à la lecture/écriture tout en incluant l'évidence qu'il n'y a de mode écrit opérationnel qu'à partir d'une langue plus ou moins connue par celui qui lit ou écrit (puisqu'aucune des langues envisagées ici ne s'écrit de façon idéogrammatique). 
société qui aura retenu notre attention dans cette étude de cas, et ce à partir de l'élément dynamique que peut constituer l'essor de la langue maternelle à l'écrit.

Pour mieux comprendre les réalités sociologiques locales et les enjeux liés à l'écriture de ces langues, s'imposait à nous de passer tout d'abord par l'outil du recensement jamais développé jusque-là sur le terrain dans le cadre d'une étude des trois «filières » ou «systèmes » éducatifs en jeu. Grâce à ces recensements, seuls des chiffres, malgré leurs limites multiples, pouvaient nous permettre de cerner les véritables rapports de proportions, voire les rapports de force entre les lettrismes locaux, au-delà des discours plus ou moins partisans des uns et des autres en faveur de tel ou tel type de savoir ou de culture. Cette approche ${ }^{6}$, à l'échelle de deux villages sénégalais ${ }^{7}$ (Juude Jaabe et Dungel) fut doublée d'une approche qualitative plus approfondie empruntant à la recherche anthropologique, sociologique, cartographique, historique et aux méthodes actives de recherche participative (MARP).

Si cette recherche, dont l'exploitation des données recueillies est en cours, permet d'ores et déjà de révéler ou de confirmer des phénomènes sociaux nouveaux pour cette société et importants ${ }^{8}$, phénomènes qu'il faudrait suivre tant ils sont porteurs de changements déjà tangibles ou potentiels, nous avons plutôt choisi de souligner ici d'autres aspects de notre enquête.

6. Effectuée par le biais de recensements relativement exhaustifs des populations avec des données notamment liées aux compétences écrites et aux filières éducatives suivies, elle a constitué également un mode de repérage efficace des individus et des entités familiales ayant un intérêt particulier — singularités ou traits communs - pour l'approche qualitative. Différents types d'acteurs ou d'entités sociales intéressants pour notre enquête ont également été mis en relief par ce biais du recensement.

7. Il eut été vivement souhaitable de mener notre travail de terrain en y incluant des villages mauritaniens, tant les réalités socioculturelles sont étroitement liées d'une rive à l'autre, malgré la frontière officielle et artificielle que constitue le fleuve entre territoire de la République islamique de Mauritanie et territoire sénégalais. Le choix de nos villages d'enquête avait d'ailleurs été déterminé, entre autres par le critère de «village-scindé » ou «villages-jumeaux », c'est-à-dire que chaque village était réparti en deux entités géographiques se faisant face sur chacune des rives. Malheureusement, malgré des accords officiels avec les autorités universitaires mauritaniennes, la police locale n'a pas jugé bon de nous laisser poursuivre nos travaux dans les villages mauritaniens retenus, pour des raisons que l'on peut imaginer dans cette zone où les relations entre autorités maures et populations haalpulaar'en ont été et demeurent plus que difficiles.

8. Évoquons par exemple une rupture entre générations, lisible à travers les choix liés aux parcours éducatifs, ou encore une répartition des individus dans les filières de savoirs selon leur sexe, leur âge et leur groupe statutaire qui connaît une certaine mutation à la fois progressive et sensible. Pour en savoir plus, voir dans quelques mois notre thèse reprenant l'ensemble des résultats et conclusions de la recherche introduite ici (sous la direction de Jean-Loup Amselle, Laboratoire de sciences sociales, EHESS-ENS). 
Dans un premier temps, il s'agira de montrer l'existence et la diversité des liens que nous avons pu observer entre les différents lettrismes - arabocoranique, français et pulaar - ainsi que leurs effets sociaux et scripturolinguistiques. L'intérêt d'une telle focalisation réside dans le fait que, communément, les acteurs ou décideurs en matière de politique linguistique et d'éducation ont tendance soit à penser les différentes langues écrites comme étant sans liens entre elles, outre celui qu'un même individu puisse avoir la maîtrise de plusieurs langues et/ou graphies à la fois, soit à les considérer dans des rapports de concurrence qui représenteraient des menaces potentielles pour les unes ou les autres, reproduisant ainsi un rapport à l'Autre (l'autre langue, l'autre culture) fondé sur la méfiance ou le rapport de force posé a priori. Puis, nous insisterons sur le caractère fluctuant de l'écriture de « nos » trois langues sur un plan diachronique, mettant ainsi en évidence les réactions ou les stratégies sociales à l'œuvre dans la progression, la régression ou la mutation des filières éducatives. Nous tenterons enfin d'associer à chaque compétence linguistico-scripturale qui nous intéresse les valeurs concrètes ou symboliques qui leur sont liées et qui sont autant d'indices sur les priorités que la société haalpulaar se fixe à elle-même.

\section{Passerelles et interactions}

Envisageons donc tout d'abord l'ensemble des liens qui peuvent s'établir entre l'arabe coranique, le français et le pulaar ou entre leurs apprentissages ou leurs acquis. Précisons que ces liens sont de natures variées et peuvent se résumer aussi bien en termes de passerelle qu'une langue propose vers une autre, ou de concurrence probable mais non prouvée, qu'en termes de liens interactifs dont les effets sont plus ou moins constructifs ou déstabilisants soit pour l'individu ou le groupe, soit pour la langue ou sa filière éducative. Nous irons des liens les plus connus ou les plus simples à appréhender vers ceux qui sont plus inattendus ou plus diffus socialement. Six relations sont ainsi à traiter : chacune des trois langues écrites vis-à-vis des deux autres.

Entre le pulaar et le français

\section{Lien mécanique à effet positif}

L'alphabétisation en pulaar a de multiples avantages qui ne sont pas propres à cette langue mais communs à tous les apprentissages de la lecture/écriture dans la langue maternelle de l'apprenant. Sur le plan pédagogique, les spécialistes s'accordent depuis longtemps à dire qu'il est incomparablement plus facile, rapide et efficace d'apprendre à lire et à écrire dans la langue 
maternelle du sujet, notamment lorsqu'il s'agit d'enfants, mais aussi dans tous les cas où la langue maternelle est mieux maîtrisée que les autres langues, ce qui est le cas chez la majorité des individus, surtout en milieu rural. Les personnes alphabétisées dans leur langue maternelle, une fois les principes de la lecture et de l'écriture acquis, et notamment les équivalences entre phonèmes et morphèmes, ont nettement plus de facultés à apprendre le français par le canal de l'écrit. Les quelques caractères qui diffèrent entre l'alphabet pulaar (caractères latins adaptés) et l'alphabet français ne posent pas de réelles difficultés aux apprenants, y compris autodidactes. On peut comparer la différence alphabétique entre les deux langues à celle qui peut exister entre le français et l'espagnol ou l'allemand. Le sujet arrive assez rapidement à faire le lien entre les caractères qu'il ne connaît pas en français pour cause d'inexistence dans sa langue et les phonèmes qui lui sont étrangers (comme le $z$, le $x$ ou le $v$ ). Grâce à l'utilisation quasi exclusive d'un alphabet latin adapté ou abajada, les personnes alphabétisées en pulaar qui, le plus souvent, n'ont pas eu accès à l'école publique, ou si peu que leur niveau est resté plus ou moins nul, trouvent donc la possibilité d'accéder assez aisément à la langue française écrite, dont ils peuvent d'ailleurs ne pas connaître les signifiés au moment de la lecture/écriture. Étant donné que l'alphabétisation en pulaar s'adresse aux adultes ou aux jeunes mais jamais aux enfants, ce type de relation entre pulaar et français concerne plutôt des migrants qui, à Dakar ou à l'étranger, vont apprendre plus facilement une langue en y ayant accès par le mode écrit et ainsi s'adapteront d'autant mieux à un environnement nouveau.

Soulignons ici que ce type de lien mécanique qui permet de passer de sa langue maternelle à l'apprentissage d'une autre langue utilisant la même graphie, ou presque, ne se limite pas, bien entendu, au lien pulaar-français. Toutes les langues occidentales partageant cette graphie sont également concernées. Il n'est d'ailleurs sans doute pas anodin que ce lien pulaarfrançais, grâce aux caractères latins, soit utilisé comme argument en faveur de l'alphabétisation en pulaar par des intervenants du secteur non francophones ou non français lorsqu'ils s'adressent à des Français ${ }^{9}$. Cela permet d'une part d'associer les acteurs français à la défense de l'usage de la graphie de Bamako face à l'ajami (graphie arabe du pulaar), d'autre part, et grâce à cette graphie latine, de donner justement accès à d'autres langues occidentales que le français.

Cette ouverture ou passerelle — potentielle et de fait — que permet l'alphabétisation en pulaar vers l'ensemble des langues occidentales pourrait contribuer à expliquer le fait que les décideurs de la francophonie, et plus encore de la politique culturelle française à l'étranger, ne participent pas à l'appui aux langues maternelles, y compris dans le domaine de l'éducation

9. Ce qui n'ôte rien au bien-fondé de l'argumentation «alphabétisation en langue maternelle d'abord pour un meilleur apprentissage des autres langues, en l'occurrence le français ». 
de base. La concurrence entre pays occidentaux dans les pays africains anciennement colonisés par la France est certainement un motif essentiel de cette politique française de «neutralité » vis-à-vis des langues nationales. « On » considère en effet que la France n'a pas à s'immiscer dans la politique linguistique sénégalaise, d'autant que le Sénégal n'a jusqu'à présent pas réellement engagé d'actions qui privilégient clairement les langues dites pourtant «nationales » dans l'éducation ou encore dans l'administration. Mais, derrière cette explication s'appuyant sur le respect de la souveraineté nationale sénégalaise en matière d'orientation linguistique, on imagine que certains préfèrent voir le français continuer à être directement acquis par les élèves plutôt qu'une langue maternelle maîtrisée à l'écrit qui donne ensuite le choix aux apprenants d'apprendre l'anglais, l'allemand, l'espagnol ou l'italien sans passer nécessairement par la langue française. Or, c'est précisément cette absence de la France sur le terrain des langues nationales qui est mal vécue par les principaux concernés, comme une négation de leurs droits linguistiques, de leur identité et de leurs valeurs culturelles au sein desquelles la langue tient une place primordiale, ce qui les détourne finalement de leurs bons sentiments ou de leur ouverture à l'égard de tout ce qui vient de l'hexagone. On peut se demander si cette attitude défensive franco-française pourra tenir encore longtemps face aux initiatives américaines, allemandes, canadiennes ou encore japonaises, s'interroger sur ses conséquences pour la francophonie et pour les pays africains concernés et chercher d'autres causes possibles qui viendraient l'expliquer comme, par exemple, la perpétuation sans doute inconsciente d'une logique coloniale d'assimilation culturelle.

À l'heure où de plus en plus de migrants originaires du Fuuta Tooro choisissent de s'expatrier vers les États-Unis ou l'Italie, mais où la France n'est pas encore exclue de la course malgré sa politique de forte limitation des entrées de migrants africains sur son sol, par ailleurs justifiée par des enjeux de politique intérieure évidents, il serait sans doute temps d'examiner ces questions de plus près en considérant l'importance que revêt l'action en matière d'éducation en langues nationales ou de soutien à celles-ci, non seulement sous l'angle de l'apport de compétences et de savoirs, mais aussi sous celui de la sensibilité des populations africaines liée à la considération que l'étranger manifeste vis-à-vis de leurs langues.

Entre le français et le pulaar

\section{Lien mécanique à effet positif}

Le lien mécanique est ici le même, en sens inverse, que celui décrit précédemment. Parmi tous les fulanisants ${ }^{10}$ croisés depuis le début de nos

10. Terme construit sur la racine «ful» (fula en anglais, fulfulde dans les pays non occidentaux de la bande soudano-sahélienne où la langue peule est usitée). Nous désignons par ce terme les personnes ayant une compétence en pulaar, non seulement orale mais aussi écrite - quel que soit le niveau de cette dernière. 
recherches en 1996, on trouve un certain nombre de personnes qui sont autodidactes en pulaar écrit. Ayant suivi un cursus scolaire, elles ont appris plus ou moins seules à lire et à écrire le pulaar par l'observation et parfois sans vraiment connaître les règles de transcription, d'orthographe ou de grammaire en usage, règles qui d'ailleurs ne sont toujours pas totalement unifiées ou unifiables, notamment en raison de la diversité dialectale à l'intérieur ou par-delà les frontières nationales.

Observons que là encore la connaissance d'une graphie ne se limite bien évidemment pas à la langue qui a servi de support à son apprentissage : en même temps que le français a pu permettre d'accéder au pulaar sous sa forme écrite, il permettait aussi à quelques-uns de développer leur usage du wolof sous la même forme. Mais cette fonction de passerelle à double détente français $\rightarrow$ pulaar $\rightarrow$ wolof semble plutôt rare, et ce pour les deux raisons suivantes. Premièrement les personnes qui cherchent, à partir de leurs compétences scripturales en français, à maîtriser le pulaar à l'écrit sont généralement motivées par des questions identitaires, culturelles ou idéologiques plus ou moins en réaction à l'expansion du wolof comme langue de communication à travers tout le pays, et ne sont donc pas très enclines à wolofiser leurs activités de lecture ou d'écriture. Deuxièmement, la production écrite en wolof, bien que cette langue soit de loin la plus parlée en tant que lingua franca, est nettement plus limitée que celle du pulaar (Diop et al. 1990).

\section{Lien matériel à effet positif}

Le second lien est d'ordre matériel et représente un atout non négligeable pour la mise en place et le bon déroulement des cours d'alphabétisation en pulaar. Il s'agit du fait que les écoles publiques mettent leurs salles de classe à la disposition des cours d'alphabétisation lorsque ceux-ci sont organisés dans le cadre de programmes gouvernementaux supervisés par la Direction de l'alphabétisation et de l'éducation de base. Il peut même arriver que cette coopération s'instaure pour appuyer des activités d'alphabétisation spontanées, que celles-ci soient prises en charge par les villageois ou financées par des ONG. Dans les deux villages étudiés, il est intéressant à cet endroit de constater que les directeurs d'école ${ }^{11}$ étaient tous deux originaires du village ou de la zone où ils étaient en poste et se sentaient donc nécessairement concernés par la vie du village et par les revendications identitaires de leur communauté auxquelles ils semblaient adhérer pleinement. L'intérêt de ce mode de fonctionnement est que cela permet aux alphabétisés de bénéficier d'un lieu relativement neutre sur le plan social. Tous peuvent s'y

11. Nous rendons ici hommage à Haruna Aliu Bubu Gaye, directeur de l'école de Dungel, qui est décédé courant février 2001, lors de notre dernier séjour sur place. Â chacune de nos missions, il avait activement participé à notre enquête et s'était beaucoup impliqué pour le développement de l'école du village. 
rendre sans distinction de sexe, d'âge ou de groupe statutaire et sans être gênés ou redevables envers telle ou telle personne ou famille qui aurait mis à disposition un lieu lui étant propre.

La limite à ce partage des locaux est que les cours de pulaar sont toujours dispensés pendant les heures creuses de l'enseignement publique, c'està-dire en soirée ou pendant les vacances, notamment les grandes vacances. Ce qui, non seulement limite les possibilités d'aménagement des horaires de cours, mais, aussi et surtout, peut poser des problèmes de luminosité, car les cours donnés en soirée dans une zone sur laquelle la fée électricité n'a pas encore jugé bon de se pencher deviennent un véritable défi aux apprenants pour distinguer avec les lampes à huile ce qui peut bien être écrit sur le lointain tableau noir. Certains d'entre eux, d'ailleurs, finissent par abandonner les cours d'alphabétisation pour de simples questions de visibilité.

\section{Lien cognitif à effets psychologique et identitaire négatifs}

Le dernier lien entre français écrit et pulaar est un lien ayant des impacts préjudiciables. Il s'agit en effet du fait que, dans le système d'enseignement public, les langues maternelles sont tellement proscrites - y compris dans les toutes premières années du cycle primaire - que l'élève, face aux difficultés de compréhension naturelles qu'il rencontre, se trouve de plus confronté, consciemment ou non, à une question de fond concernant l'aptitude de sa langue à être l'objet ou le vecteur d'un savoir ou de compétences intellectuelles - question qu'il peut se poser en admettant qu'il ne soit pas déjà persuadé que la réponse est négative. Derrière ce doute ou cette vision limitative de la langue maternelle se profile simultanément la question d'une hiérarchie des langues ou celle d'une répartition de leurs vocations/aptitudes selon les usages visés, hiérarchie ou répartition au sein desquelles le pulaar serait forcément déprécié. Et étant donné qu'intuitivement, langue, culture et identité sont étroitement liées, il en découle un malaise identitaire difficile à dissiper par la suite tant il aura été introduit à un jeune âge, que des mots auront rarement permis à l'enfant de le mettre à distance et de le comprendre, et que la pression en classe pour que l'enfant n'utilise pas le pulaar est omniprésente et distillée dans un contexte où la chicotte est toujours en pratique, bien qu'interdite dans les textes.

Cela dit, il est intéressant de constater que certains maîtres pulaarophones assouplissent les directives nationales et ont parfois recours à la langue peule pour dénouer des zones d'incompréhension partagée par l'ensemble des élèves, ce qui se révèle efficace ${ }^{12}$. Il y a là une certaine évolution, même légère, des pratiques des enseignants tant cela était inconcevable

12. Il serait intéressant de savoir si ces interventions en langue maternelle peule se pratiquent également dans d'autres milieux ruraux à dominante monolingue, par exemple en milieux jola, sereer ou mandinka. La réponse enrichirait la lecture que l'on peut avoir de l'ampleur et de la singularité du mouvement pulaar qui se fonde sur une conscience identitaire forte et des pratiques qui la manifeste et qui pourraient transparaître chez les maîtres haalpulaar'en. 
au cours des décennies passées. Il n'est pas impossible à cet égard que le processus de décentralisation engagé par l'État il y a quelques années ait participé de façon diffuse à ces petites prises de liberté de certains maîtres.

\section{Entre l'arabe coranique et le français}

\section{Lien de transfert et de valorisation indirecte}

En milieu haalpulaar, l'école coranique est aujourd'hui quasiment devenue un passage obligé pour tout enfant, qu'il soit ou non destiné par ses parents à une scolarisation formelle. Mais, aujourd'hui sans doute plus qu'hier, les enfants quittent d'eux-mêmes le dudal ${ }^{13}$, officiellement parce qu'ils «n'ont pas la tête faite pour l'étude », qu'ils ne sont pas intelligents ou qu'ils sont distraits, ne pensant qu'à s'amuser - discours tenu aussi bien par les parents que par les enfants ou les jeunes concernés. En réalité, et presque systématiquement, en creusant le non-dit, on se trouve face à des situations de mauvais traitements corporels de la part des marabouts sur les enfants confiés. Cela ne choque d'ailleurs personne tant il est ancré que pour bien apprendre il faut «être corrigé » dans tous les sens du terme. Les parents, y compris ceux-là même qui avaient abandonné l'école coranique pour ce genre de motifs, donnent donc généralement raison aux pratiques de la majorité des marabouts.

L'effet de cette liberté que prennent les enfants et qu'ils imposent à leurs parents en quittant d'eux-mêmes leur dudal, souvent à travers un rapport de force très dur quand le père a de plus grandes ambitions de formation ou de carrière religieuse pour son enfant, est qu'ils redeviennent disponibles pour d'autres activités. Il est fort probable que les enfants qui quittent d'euxmêmes leur marabout le font dans la perspective ou l'espoir de pouvoir suivre les cours de l'école par la suite, comme le font une bonne partie de leurs frères et sœurs aînés ou de leurs compagnons de classe d'âge ou de ceux de la classe d'âge aînée. Quoi qu'il en soit, et que leur abandon de l'école coranique soit impulsé par eux seuls ou avec le consentement ou la décision de leurs parents, deux voies s'offrent alors aux ex-'almubbe : soutenir la famille par une activité lucrative, alimentaire ou domestique quelconque ou éventuellement, donc, se réorienter sur une filière éducative plus valorisée à leurs yeux : l'école du village, s'ils n'ont pas encore atteint la limite d'âge. Il nous est difficile pour l'instant de trancher en faveur ou en défaveur d'un lien de cause à effet entre l'abandon de l'école coranique en

13. Signifie «foyer» en pulaar. L'apprentissage du Coran s'effectue fréquemment de nuit, autour d'un feu qui réunit les jeunes disciples le soir, après les travaux champêtres ou l'aumône, et avant le lever du jour et la première prière quotidienne. À ces moments-là, l'air est souvent frais et le feu autour duquel sont réunis les almubbe a la fonction non seulement d'éclairer les tablettes de bois sur lesquelles sont inscrits les versets à mémoriser mais aussi de les réchauffer. 
bas âge et le recrutement en progression dans les écoles publiques, car d'autres facteurs, que nous ne pouvons développer ici, peuvent venir expliquer la «baisse » des effectifs de l'une et l'augmentation de ceux des autres. Cependant, un tel lien est tout de même fort probable, ne serait-ce que par l'effet de mimétisme social qui s'opère en matière de choix éducatifs, malgré le principe de complémentarité qui caractérise les fondements de la société haalpulaar comme ceux de nombreuses sociétés africaines.

Notre travail à Dungel et à Juude nous a par ailleurs permis de relever un phénomène psychologique récurrent chez les enfants qui ne vont qu'à l'école coranique. Nous nommerons ce phénomène «le complexe de l'"almuudo ». En effet, que l'attitude extérieure soit de l'ordre de l'introversion excessive et de la gêne face à l'observation ou à des questions personnelles, ou au contraire de celui de l'arrogance et d'une assurance défiante vis-à-vis des adultes, on finit presque toujours par s'apercevoir que la participation à l'enseignement du marabout n'est pas un choix de l'enfant, ni même un réel consentement, mais plutôt un compromis que l'enfant accepte lorsque les pressions affectives, matérielles ou physiques se font trop fortes. Et l'enfant d'envier secrètement ses camarades scolarisés, et souvent de tenter d'apprendre par eux-mêmes dans les livres de ces derniers, ou en même temps qu'eux, lors de leurs devoirs quotidiens. Il semble que ce n'est que plus âgés que certains, qui persévèrent dans l'étude du Livre Saint, revendiquent leur formation religieuse, comme si un retournement — psychologique ou spirituel — s'était opéré en eux, peut-être au moment où ils acceptent l'idée d'une «carrière » religieuse.

Et pour comprendre que ce complexe ou cette frustration de l'enfant ou du jeune qui n'a pas accès à l'école publique ${ }^{14}$ fasse l'objet d'une certaine dissimulation de la part des jeunes restés à l'école coranique, il suffit de penser qu'un enfant, en Afrique, ne doit pas contrarier la volonté parentale, sous peine d'être frappé — physiquement ou de malédiction — ou délaissé affectivement. De plus, la situation matérielle de la famille ne laisse pas toujours le choix aux parents et aux enfants quant à leur «placement» auprès d'un marabout qui ne demande pas d'argent et fait en sorte que les enfants soient plus ou moins nourris par l'aumône qui leur est faite ou par le fruit de leurs travaux champêtres. Enfin, la volonté de Dieu est invoquée par les parents dans ce choix d'éducation coranique. Tout musulman doit assurer à ses enfants la possibilité de prier Dieu, et donc de connaître au minimum les sourates et prières de base, ce qui passe systématiquement par l'école coranique et la prise en charge de l'enfant par un marabout. Et les

14. Sont aussi concernés les enfants qui ne suivent aucune filière éducative, plus encore peut-être que ceux qui vont seulement à l'école coranique et qui, même s'ils ne l'ont pas choisi de leur plein gré, engrangent un savoir et ont une position sociale «positive », c'est-à-dire qu'ils ne sont pas seulement bons à aider la famille à subsister, mais qu'on leur a destiné un certain parcours qui doit leur être profitable personnellement, même si ce dernier est très éprouvant moralement et physiquement. 
enfants savent bien cela et ne peuvent pas en disconvenir ouvertement; ce serait renier Dieu d'une certaine façon. Chez ceux qui acceptent donc de vivre la vie d' 'almuudo et de rester dans cette voie religieuse, l'amalgame a joué entre volonté parentale, pression sociale des aïeux et recommandations ou obligations divino-religieuses.

Entre le français et l'arabe coranique

\section{Lien concurrentiel et/ou stimulant à effet pédagogique positif}

Revenons un instant à la question de la violence ou des punitions corporelles exercées par la plupart des marabouts sur leurs jeunes disciples. Face à la désertion de leur duqal ou à la baisse de leurs effectifs, et sans doute aussi sous l'influence de l'école publique qui se veut moins violente physiquement et vers laquelle se (re)tournent parents et enfants, certains marabouts revoient leurs approches «pédagogiques » et tentent d'enrayer leurs habitudes vivement démonstratives en cas d'erreurs ou d'inattentions de la part de leurs 'almubbe. Malheureusement, bien peu de mesures sont prises à notre connaissance au Sénégal en matière de réflexion et d'accompagnement en direction de l'enseignement coranique informel, notamment sur le plan pédagogique. Et lorsque support il y a, il vient surtout d'ONG américaines ou canadiennes (Mbacke 1994: 78) et ne semble pas avoir porté ses fruits dans la zone qui nous intéresse. Chacun bricole donc ses propres méthodes.

Dans cette lente évolution du mode de relation entre enseignant et enseigné de la filière coranique, interrogeons-nous sur le fait qu'apparemment les parents ne soient pas partie prenante dans ce processus de réduction de la violence et assument mieux que les marabouts cette réalité du châtiment corporel comme partie intégrante de l'enseignement. Tout d'abord, il est évident que les parents, n'étant pas les auteurs de l'acte, s'en sentent moins responsables ou «fautifs» aux yeux des approches occidentales actuelles. Ensuite, lorsqu' on confie un enfant à un parent ou à quelqu'un qui est chargé de l'instruire, la bienséance (ou la négligence, voire la lâcheté ?) veut qu'on ne se mêle pas — à plus forte raison lorsqu'il s'agit d'un représentant du religieux auquel le sacré est identifié —, même en tant que père ou mère, de la façon dont l'adulte se conduit, lui qui par définition est plus âgé que l'enfant confié et donc dans son bon droit. Enfin, et plus prosaïquement, les moyens de subsistance des marabouts sont le plus souvent concentrés sur leurs activités de «maître coranique ». Même si souvent les marabouts doivent assurer l'entretien du jeune disciple, ce dernier rapporte plus qu'il ne coûte : il quémande des restes de nourriture pour manger, de l'argent ou tout autre denrée, cultive les champs du marabout et les parents peuvent participer selon leurs moyens, financièrement ou par des dons en nature. Le marabout a donc intérêt à avoir le plus d' 'almubbe possible afin d'assurer sa propre subsistance. Or pour accroître le 
nombre de ses recrues et fidéliser celles-ci, le maître doit anticiper sur le risque de leur abandon/fuite du cercle coranique et tenter de trouver un compromis entre l'image, encore largement associée à la fonction de maître coranique, d'une sévérité rude mais bénéfique à l'enfant, sévérité à laquelle certains parents restent très attachés, et la nécessité de réviser leur attitude vis-à-vis des châtiments corporels employés sur des enfants qui, par la suite, refuseront de fréquenter le maître malgré une éventuelle pression parentale. Des pratiques pédagogiques plus souples et moins traumatisantes physiquement pour le jeune « apprenti coranique » correspondent donc à une adaptation du marabout face à des taux de fréquentation plus fluctuants ou plus bas qu'auparavant qui menacent directement la situation matérielle et financière du maître.

Reste à se demander quelles incidences peut avoir cette évolution pédagogique a priori positive en termes de maintien d'une figure d'autorité structurante dans l'éducation des jeunes, et ce en l'absence de beaucoup de pères de famille en migration? L'évolution des mœurs de nombreux enfants et adolescents, qui même au village s'opposent parfois violemment aux mâ̂tresses de maison et agissent en défiant tous les repères et valeurs « traditionnels », ne songeant qu'à quitter leur village, ou leur pays, laissent tout un champ d'investigations et d'hypothèses ouvert...

\section{Lien hypothétique à effet social et religieux dynamique}

À l'image des cours de pulaar qui se tiennent généralement lors des périodes creuses de l'enseignement formel, les effectifs des dude augmentent sensiblement le soir après l'école ou en période de vacances scolaires. Cela a pour effet de développer le caractère saisonnier de l'activité maraboutique, de provoquer des reconversions d'une partie d'entre eux à certaines périodes de leur existence où ils n'ont plus d'élèves, ou d'encourager chez eux une logique de démultiplication de leurs activités, mystico-religieuses ou non. On observe notamment un phénomène assez récent de «navettisation » de leurs activités mystiques ou «travaux »: à intervalles relativement réguliers, les marabouts, qui ne sont pas imams en même temps, vont faire des séjours de quelques semaines à Dakar pour avoir de nouveaux débouchés dans le domaine de leurs services mystiques et renflouer ainsi leurs fonds. Ils « recrutent » leurs nouveaux clients dans les cercles familiaux et relationnels de leur parents implantés dans la région du Cap Vert (Dakar et ses banlieues populaires). Ils tentent aussi de développer ce genre de services à distance, par le courrier postal et éventuellement le téléphone qui se trouve à la souspréfecture mais dont l'accès n'est pas facile (coûteux et éprouvant physiquement à cause des trajets en charrette sur des pistes non entretenues).

Sur le plan social, on observe une forte démocratisation de l'enseignement coranique. Autrefois réservées aux rimbe (hommes libres ou nobles), les concessions des marabouts sont aujourd'hui fréquentées par des enfants 
issus de tous les groupes statutaires (nobles, artisans « castés » ou esclaves). Après une première période de résistance à la scolarisation des fils de nobles dans les années 1950-1960, les couches aristocratiques villageoises ont ensuite changé leur fusil d'épaule en voyant que les fils d'esclaves envoyés à la place des leurs avaient finalement eu des positions socioprofessionnelles enviables. Et chacun d'envoyer ensuite son fils à l'école, ce qui diminuait les effectifs des cours coraniques. On peut penser que cette ouverture de la filière religieuse a correspondu à ce phénomène de changement de stratégie éducative des familles libres. Les clivages sociaux ont sauté puisque les nobles eux-mêmes ont «décoranisé » — partiellement, certes — leur descendance. Cette démocratisation de la filière coranique pose plusieurs questions de fond. Les motivations des familles non nobles sont-elles seulement de type religieux ? Quels sont les enjeux d'autres motivations possibles, comme, par exemple, la motivation de prise de pouvoir ou d'influence, de vengeance des dominés sur les dominants ? Quelles peuvent être les conséquences de ce phénomène sur l'évolution de l'islam en tant que science religieuse et mystique, en tant que pratique pour l'ensemble des populations villageoises et enfin en tant que sphère de pouvoir au sein de la société haalpulaar?

\section{Entre I'arabe coranique et le pulaar}

\section{Lien idéologico-religieux à effet plus ou moins positif}

Le lien ici est tout à fait indirect, mais néanmoins important. Il s'agit de l'attitude favorable, voire engagée vis-à-vis du pulaar, de marabouts fuutanke liés aux autorités religieuses et à la mouvance de Madina Gounas ${ }^{15}$. Nous avons ainsi croisé des marabouts qui ont suivi tout un cursus en pulaar en ville, cursus parfois assez poussé, en même temps qu'ils faisaient leurs premières armes en tant que maître coranique ou qu'ils travaillaient dans d'autres domaines à des fins lucratives. Une fois rentrés dans leur village d'origine, n'ayant pas forcément entretenu leurs compétences écrites en pulaar ou ne les intégrant pas dans leurs enseignements islamiques, il ne reste souvent de leur expérience fulanisante qu'une bonne disposition à l'égard des activités villageoises d'alphabétisation en pulaar. Ce qui en soi est déjà beaucoup, car si la position des marabouts vis-à-vis des initiatives renforçant la langue maternelle était défavorable, on pourrait penser non seulement que ces dernières sont vécues comme une menace pour l'islam local, mais aussi que le mouvement pulaar aurait trouvé là un frein considérable en la personne des marabouts.

15. Petite ville située dans la région de Kolda, au Fouladou, devenue le fief d'un islam tidjane militant pour la cause pulaar, la langue et la culture des Peuls et Haalpulaar'en. 
Même chez les marabouts qui ne sont pas liés à Madina Gounas, on note une attitude plutôt encourageante pour les initiatives liées à la langue et à la culture peules, dont ils sont d'ailleurs, comme tout autre villageois, des représentants à part entière indépendamment de leurs fonctions ou de leur statut religieux. Mais à côté de ces positionnements favorables, on en trouve d'autres plus neutres ou plus gênés de la part des représentants locaux de l'islam, comme si la démocratisation de l'accès au savoir, toutes filières éducatives confondues, remettait en cause les équilibres sociaux et représentait un danger potentiel ou déjà palpable pour une partie de l'élite statutaire — dont les toorob6e — à laquelle les attributions religieuses étaient par principe réservées.

Entre le pulaar et l'arabe coranique

\section{Lien psychologique à effet religieux positif}

Un résultat surprenant de notre enquête est que le pulaar maîtrisé à l'écrit sert de support à un apprentissage oral du Coran. Quand l'écrit se met au service de l'oralité...

Lorsqu'on interroge des néo-alphabètes en pulaar sur ce que leur a apporté la pratique écrite de/dans leur langue, on trouve systématiquement des réponses du type «cela m'a ouvert les yeux sur le monde », «avant, j'étais endormi, maintenant, je suis éveillé », «cela m'a donné plus confiance en moi, m'a permis de faire telle ou telle activité ${ }^{16}$. C'est cette confiance en soi qui permettrait aux fulanisants adultes d'oser aller trouver un marabout pour le solliciter, alors qu'ils ont dépassé l'âge où l'on s'attelle traditionnellement à ces apprentissages. En fait, aller voir le marabout pour lui demander de réciter les versets permettant de prier est en soi un genre de confession : "Avant aujourd'hui, je ne savais pas vraiment prier, et ne priais donc probablement pas, ou pas convenablement, et j'ai passé toute ma vie ainsi. » Pour dépasser cette inhibition, le fait d'être déjà passé, dans une autre langue, par l'expérience du type «c'est possible, à tout âge » et « voilà qui j'étais avant d'être alphabétisé(e), et voici qui je suis devenu(e) grâce à l'enseignement suivi » est très certainement déterminant pour ceux qui font le pas vers un marabout.

Au-delà de ce sentiment de honte, prendre note tout seul sous la dictée d'un marabout qui ne vous a pas appris à lire et écrire est en même temps une fierté personnelle et un signe d'ascension sociale. Et si l'on a plutôt tendance à voiler cette fierté dans une société où les sentiments doivent

16. Le croisement des données recueillies à travers plusieurs enquêtes et évaluation, dont l'excellente évaluation d'ARED (ARED 2000) sur un de leur programme d'alphabétisation en milieu pastoral peul, permet de valider totalement les données que nous avons recueillies sur ce point dès notre premier terrain pour notre mémoire de DEA en 1996. 
être contrôlés dans leur manifestation et où la pudeur (semteende) et la modestie constituent en partie le modèle comportemental à suivre (la fameuse pulaaku), elle n'en est certainement pas moins ressentie par l'individu. D'autant que par ses acquis antérieurs en pulaar, il va pouvoir dédoubler ses connaissances, et ce dans un domaine des plus appréciés par l'ensemble des villageois, et notamment par les nobles.

Sur cette passerelle intéressante que propose le pulaar vers l'arabe coranique, on peut affirmer que le fait de pouvoir noter les versets coraniques à l'aide de la graphie latine du pulaar (légèrement adaptée pour transcrire l'arabe), ôte la barrière de l'apprentissage d'une seconde graphie, celle de l'écriture arabe, que certains alphabétisés n'auraient probablement pas le courage de franchir après avoir déjà fourni les efforts d'intégration d'un premier alphabet ${ }^{17}$.

Nous avons donc affaire ici à une transversalité à tous les niveaux : entre filières éducatives et entre les langues qu'elles enseignent qui de plus, sont transcrites dans deux graphies différentes, et enfin transversalité entre mode écrit et mode oral, puisque l'écrit est utilisé pour mémoriser un texte fait pour être « récité »(prié oralement).

Sur le plan social, il y a à la fois bénéfice et danger si ce type d'usage transversal se généralise. Bénéfice car cela élargit l'accès à davantage de connaissances et de possibilités de cheminer de façon autonome sur le plan de la vie spirituelle et de la pratique religieuse des individus. Danger car si cette pratique d'utilisation de l'alphabet pulaar pour apprendre le Coran devient plus large, on peut se demander si cela n'encouragera pas à terme à un abandon de l'apprentissage coranique «traditionnel », c'est-à-dire via le mode écrit en caractères arabes. Se profile alors la mise en péril de l'enseignement et des sciences coraniques et islamiques en tant que statut social et sphère d'activité professionnelle dont l'aura repose en partie sur cet usage de la graphie arabe qui participe à la sacralité et à l'identité de l'islam tel que son image est constituée aux yeux des croyants. Ou bien cela donnera encore davantage de pouvoir religieux à ceux, de moins en moins nombreux, possédant encore les connaissances scripturales arabes néanmoins pour accéder aux textes «savants » de l'islam.

Mais nous sommes dans la fiction et les quelques fulanisants qui ont cette démarche d'apprentissage du Coran, grâce à leurs acquis en pulaar, représentent pour l'instant une minorité qui a bien peu de chance de prendre un poids considérable pouvant influencer la filière coranique, car les fulanisants sont encore peu nombreux comparativement à l'ensemble des populations des deux villages étudiés (environ $8 \%$, tous niveaux confondus), ou

17. Signalons d'ailleurs ici la diffusion au Sénégal de nombreux petits livres ou brochures relatifs à l'islam qui sont écrits en langue arabe avec des caractères latins. Ces livres sont parfois bilingues, surtout arabe-français, et présentent donc dans certains cas trois versions d'un texte : arabe en caractères arabes, arabe en graphie latine et traduction en français. 
même par rapport aux personnes ayant reçu ou étant en cours de formation dans les deux autres filières, «française » et coranique.

À propos de cette passerelle que permet le pulaar écrit vers l'apprentissage du Coran oral, il est important de noter que cela est propre au pulaar, et que les francisants n'ont pas la même démarche alors que, logiquement, il leur serait également possible de l'adopter. Nous voyons essentiellement deux explications à l'origine de cette barrière entre sphère francisante et apprentissage du Coran.

En termes de proximité culturelle, islam et culture haalpulaar font bon ménage depuis plusieurs siècles, sans que cette proximité n'ait vraiment connu d'épreuves de force ou de contrainte ${ }^{18}$ imposées de l'extérieur comme ce fut le cas lors de la pénétration coloniale. Alors que la maîtrise écrite en français et le français lui-même en tant que langue sont toujours assimilés à une culture occidentale par nature lointaine de la culture locale haalpulaar et islamique. Une seconde explication, d'ordre mécanique, est certainement également au cœur de la non-transversalité entre compétences écrites issues de la filière scolaire et Coran. Il s'agit du fait que presque tous les individus, surtout de sexe masculin, qui sont passés par l'école de leur village, ont quitté celui-ci pour trouver du travail dans les centres urbains du Sénégal, dans d'autres pays africains ou encore en Occident. Ils ne sont donc plus sur place pour solliciter les marabouts rencontrés à Juude ou à Dungel. Il serait donc intéressant de voir si dans les milieux de migrants haalpulaar'en une jonction s'établit entre compétences alphabétiques acquises avec l'apprentissage du français et démarche d'apprendre le Coran avec une graphie latine.

Le dernier aspect important à envisager concernant les liens entre pulaar et arabe coranique est la question de l'ajami. Depuis environ deux siècles, le pulaar s'écrit - comme d'autres langues africaines d'ailleurs, tel le hausa - avec des caractères arabes adaptés. La particularité de cette graphie arabe du pulaar est qu'elle est utilisée aujourd'hui par une partie infime de la population, à savoir par les lettrés de la filière coranique généralement âgés, en grande partie des marabouts - et encore, une partie seulement d'entre eux l'utilisent. L'ajami a une double fonction : il permet aux écrits mystiques ou magiques de ne pas livrer leurs secrets précieux et puissants aux non-arabisants non pulaarophones qui pourraient chercher à les lire, donc aux étrangers en principe, tout autant qu'il a l'avantage de préserver les connaissances personnelles du marabout, qui en font sa force et sa spécificité, y compris du regard des autres marabouts «concurrents», puisque chacun met au point sa propre codification pour transcrire voyelles et sons

18. Même la révolution des Almamy vers 1770, qui renversa le pouvoir peul des Deeniyankoobe, officiellement islamisés mais encore très imprégnés de paganisme, pour instaurer un régime théocratique, est partie de la société haalpulaar elle-même. 
du pulaar inexistants en arabe, et adapter ainsi la graphie arabe à la langue peule.

$\mathrm{Au}$ fil du temps, l'ajami semble bel et bien en perte de vitesse. Le nombre des scripteurs l'utilisant régresse de toute évidence, et lorsque des assemblées permettent d'en repérer quelques-uns, non seulement ils sont peu nombreux mais encore sont-ils âgés. Leur renouvellement n'est pas vraiment assuré, ou dans des proportions si faibles que nous n'avons rencontré aucun de leurs représentants parmi des jeunes dans nos villages. Plus encore, le mot «ajami » est quasiment toujours source d'incompréhension, ignoré qu'il est, lors d'échanges à ce sujet avec l'ensemble de nos interlocuteurs, quel que soit leur âge, à l'exception des quelques rares individus qui en ont l'usage. Et même en expliquant sa signification, on observe le plus souvent que les gens ont beaucoup de mal à comprendre ce dont il s'agit, comme si la représentation que l'ensemble des villageois, alphabétisés et analphabètes, se fait du pulaar à l'écrit excluait la réalité ou la possibilité d'une graphie arabe pour transcrire la langue maternelle. Cela est non seulement représentatif de la déliquescence actuelle que connaît cette écriture, mais aussi de l'étroitesse des cercles de lettrés qui autrefois, plus largement qu'aujourd'hui sans doute, utilisaient l'ajami ${ }^{19}$. Il y a donc eu, manifestement, une évolution dans le temps de cette pratique linguistico-scripturale, en lien avec des enjeux sociaux, politiques, culturels et religieux.

Ce qui nous mène à envisager les productions ou activités écrites au Fuuta Tooro dans les trois langues et deux graphies étudiées comme étant bien plus que des contenus insérés dans des systèmes de communication ou utilisant un mode d'expression particulier, celui de l'écrit. Il s'agit en effet également d'indicateurs emblématiques de stratégies politico-culturelles menées par les populations locales, le plus souvent tout à fait sciemment et plus ou moins sous la dictée des couches sociales et des acteurs, endogènes ou exogènes, détenant les pouvoirs de décisions ou ayant des moyens de pression efficaces.

\section{Flux et reflux des langues écrites}

Nous avons vu combien les relations entre les langues, les systèmes écrits et les réalités sociales pouvaient être dynamiques, dans une approche synchronique. Nous souhaitons à présent, sans rentrer dans les détails, montrer que ce caractère dynamique s'inscrit aussi dans une dimension temporelle et qu'il n'y a pas eu encore dans la Vallée de «choix » local stable

19. Au Fuuta Tooro, l'ajami fut relativement peu développé en comparaison d'autres régions d'Afrique de l'Ouest comme le Fuuta Jaloo. L'influence de grands marabouts fut déterminante quant à l'essor que connut cette écriture. En effet, au Fuuta Tooro, El Hadj Omar Tall aurait plaidé en faveur de l'écriture en langue arabe aux dépens de l'ajami, tandis qu'à la même époque, Ceerno Mamadou Samba Mombeya prônait au contraire vivement la production en ajami. 
dans le temps concernant une langue écrite à mettre à la portée de tous, ou du plus grand nombre. Cette dynamique diachronique peut se jouer, pour chaque langue écrite, de façon autonome par rapport aux autres scripturalités ou au contraire en lien avec celles-ci.

Commençons par la langue la plus anciennement écrite dans la région, l'arabe. Langue diplomatique et langue religieuse, ayant en outre été le support d'une poésie religieuse abondante, l'arabe coranique dans la région qui nous intéresse a longtemps été le fait d'une élite restreinte qui tirait d'ailleurs son statut élitaire de la maîtrise du Coran, des sciences développées par l'islam et donc bien sûr de l'écriture de/en arabe. Étant donné que ce groupe statutaire, qui avait renversé le pouvoir dynastique mis en place par Koli Tengella, fondait toute sa légitimité sur son identité ${ }^{20}$ et ses compétences islamiques «authentiques », par opposition aux Peuls qu'ils avaient détrônés et qui étaient des musulmans de façade, et que cette légitimité le plaçait au plus haut de la hiérarchie sociale, il n'était évidemment pas question de « démocratiser » l'accès aux dude. Ce ne serait qu'assez récemment, bien après les débuts de la colonisation française, que cet accès se serait ouvert, mais non sans lien avec l'histoire de l'école publique.

Dans le processus de colonisation, il y eut un décalage assez important entre la période d'implantation physique des Français sur le territoire de l'actuel Sénégal et la mise en place d'une politique éducative en faveur d'un enseignement plus large pour les «indigènes » concrétisée par les premières écoles ouvertes dans quelques villages de la Vallée au cours des années 1930 (à Mbummba et Kaskas). La création de ces écoles s'accompagna de l'obligation pour les villages alentours de fournir des élèves dont le recrutement était réglementé selon des critères spatiaux (par quartiers) et sociaux précis (les familles détenant le pouvoir dans chaque village). Les notables développèrent alors des stratégies pour feinter l'institution et envoyer, à la place de leur fils aîné ou de l'un de leur fils, un fils issu des familles asservies qui leur étaient liées ${ }^{21}$. À l'époque, l'enfer était promis par les marabouts à celui qui enverrait son enfant chez les Blancs qui par nature « accultureraient » et «sataniseraient » leur progéniture ${ }^{22}$, ce qui non seulement condamnait l'enfant concerné, le fils bien-aimé, à une mort symbolique pour ses parents, mais encore pouvait compromettre la reproduction de l'ordre social établi reposant sur la transmission lignagère des pouvoirs. Envoyer son enfant à l'école revenait alors au même pour les villageois

20. Le nom même de ce groupe statutaire des toorobbe ou toorodbe signifie «ceux qui louent Dieu » ou « ceux qui louent Dieu ensemble », selon plusieurs sources, dont Schmitz (1993: 18).

21. Il arrivait aussi que les enfants fuguent en complicité avec leurs parents et aillent se réfugier dans un village voisin, par exemple.

22. Plus précisément, les marabouts disaient que la main qui emmenait l'enfant dans les écoles de Tubakoobe (Blancs) serait la même main qui les conduirait demain en enfer. Cela se dit toujours, mais la mise en garde s'est banalisée. 
que de le voir partir pour être enrôlé dans l'armée française et combattre sur les fronts de la Deuxième Guerre mondiale.

Dans «nos » villages enclavés, il fallut attendre le milieu des années 1960 pour voir arriver l'institution scolaire. Dans ces années et dans la continuité de l'époque coloniale, le jeune État sénégalais se méfiait encore des écoles coraniques considérées comme « concurrençant dangereusement l'école française » (Mbacke 1994 : 80). Les deux parties se regardaient donc en chiens de faïence et cet esprit de rivalité et de méfiance réciproque était conforté par la négation mutuelle de la valeur des connaissances et des aptitudes transmises par chaque filière.

Lorsque la première génération de scolarisés atteignit l'âge adulte, certains de ses éléments connurent une réussite sociale et matérielle que les « vrais » fils de notables leur enviaient. Cette réussite allait d'ailleurs à l'encontre des objectifs que s'était fixés l'administration coloniale concernant les écoles rurales. Celles-ci devaient en effet, selon l'inspecteur de l'enseignement de l'AOF, Georges Hardy, «maintenir à la campagne, dans une situation plus heureuse, les populations qui s'y trouvent, et non faire naître chez les enfants le désir d'être fonctionnaires » (Léon 1991 : 21). Dès lors, le retournement s'opéra et ceux qui jadis étaient promis à une filière coranique complète furent scolarisés, tout en continuant pour une partie d'entre eux à suivre l'école coranique lorsqu'ils le pouvaient, en dehors des horaires scolaires. Mais pour ceux qui faisaient un double cursus, il y avait comme un accord tacite entre parents et enfants qui donnait la priorité à l'école publique, et la fréquentation du marabout n'était clairement plus à des fins de fonction religieuse au sein de la communauté villageoise. Après la première génération de descendants d'esclaves qui réussirent, et à cause de la scolarisation dans le système formel des enfants des familles toorob̧e, l'école coranique a subi une baisse de ses recrues, et c'est à cette période que la démocratisation des dude est amorcée.

Nous sommes alors dans les années 1970-1980 à la fin desquelles les régimes africains postcoloniaux commençaient à s'essouffler sérieusement, ne pouvant plus continuer à s'endetter de façon inconsidérée pour financer des fonctionnaires dont les effectifs pléthoriques alourdissaient le système étatique plus qu'ils ne débouchaient sur un développement économique et social durable. Or, c'était essentiellement dans la fonction publique qu'étaient recrutés les bacheliers sénégalais. La crise économique aidant, l'école comme l'université ne furent bientôt plus considérées que comme des voies de garage dont les frais qu'elles entraînaient ne constituaient qu'une charge non rentable, même à terme, pour la famille. Et certains continuent de penser aujourd'hui encore qu'il vaut mieux avoir un enfant qui soit «bana-bana » (commerçant ambulant) ou domestique à Dakar que de lui faire perdre son temps à aller à l'école, sacrifiant ainsi le peu de ressources familiales éventuellement disponibles.

Malgré tout, l'institution survivait dans les villages de l'Ile à Morphil qui nous intéressent, mais sans dynamisme, stagnant quasiment sur le plan 
du nombre d'élèves. Et si elle n'a pas complètement périclité, c'est peutêtre qu'il n'y avait de toute façon pas de perspectives d'avenir très réjouissantes pour les parents, et que l'on avait tendance, dans les familles nobles, à répartir sa progéniture selon les deux filières éducatives existantes et à faire travailler les enfants que l'on n'envoyait pas étudier.

C'est à ce moment-là que la Vallée connut un boum humanitaire. Après les sécheresses des années 1970 et les flux migratoires qu'elles provoquèrent, de nombreuses ONG intervinrent dans la zone. Ces associations indépendantes souvent étrangères ou para-étatiques, dont les objectifs étaient le développement local, notamment via l'agriculture irriguée, s'appuyèrent de leur plein gré ou à la demande pressante des organisations villageoises sur des programmes d'alphabétisation en pulaar. Elles s'implantèrent dans la région à une période où les militants de la «cause » haalpulaar ou peule la sillonnaient pour sensibiliser les populations sur l'importance de la survie de leur langue pour assurer celle de leur identité et de leur culture, et sur la nécessité, pour ce faire, de s'alphabétiser en pulaar.

D'abord d'ordre identitaire et culturel, le mouvement pulaar devint assez rapidement motivé par des enjeux de pouvoir économique local. Malgré les quelques attentions dont le pulaar avait été l'objet à la fin du XIX ${ }^{\mathrm{e}}$ et au début du $\mathrm{XX}^{\mathrm{e}}$ siècle de la part d'administrateurs coloniaux qui flirtaient avec l'ethnologie et la linguistique ${ }^{23}$, et n'ayant guère accès à l'ajami, la langue peule du Fuuta Tooro était auparavant parlée, vécue, mais très probablement ne pouvait-elle pas être considérée par les Fuutanke comme langue déjà écrite, ou que l'on peut écrire. Pour preuve de cette représentation par la négative de la scripturalité potentielle du pulaar, les réactions furent innombrables lors des campagnes de sensibilisation, des premières sessions d'alphabétisation et parfois encore à l'heure actuelle consistant à juger stupide d'aller à des cours où l'on apprend sa propre langue. L'amalgame entre apprendre une langue et apprendre à lire, à écrire et à manier une langue à l'écrit était bien ancré ${ }^{24}$ et il lui faudra du temps pour s'atténuer.

À ses débuts, le mouvement pulaar constitua en quelque sorte une révolution idéologique et culturelle : écritures et systèmes éducatifs avaient toujours été dans le passé le fait de langues et de cultures étrangères ; il était auparavant inconcevable de suivre un cursus d'enseignement dans sa propre langue et de pouvoir valoriser ensuite celui-ci dans son environnement quotidien ou en lien avec des institutions ou organisations nationales ou étrangères. Et si ce furent surtout les femmes et les jeunes qui s'y investirent bénévolement et y crurent les premiers, les hommes restés au village allaient le rejoindre dans des buts plutôt de valorisation de leur personne à des fins professionnelles ou en lien avec des activités lucratives (collaborer avec des ONG, gérer des périmètres irrigués privés ou villageois, devenir alphabétiseur). Il y eut donc un certain engouement pour le pulaar. Mais cela ne dura pas.

23. Il s'agit de Faidherbe, Binger, Gaden et Delafosse (Schmitz 1998: 40-41).

24. Plusieurs hypothèses explicatives seront bien sûr données à cela dans notre thèse. 
Les années 1990 furent celles de l'apothéose des activités villageoises « spontanées » liées à l'alphabétisation. Mais les premiers animateurs du mouvement dans chaque village n'y restèrent pas, appelés par d'autres activités hors du village ou par des projets migratoires et la relève se fit difficilement. Les nouveaux alphabétiseurs voulaient être payés, à l'image de ceux qui étaient recrutés par des ONG, et, malgré leurs promesses, les villageois avaient et ont toujours du mal à convaincre les personnes-ressources locales de leurs capacités à prendre en charge un petit salaire. Les programmes d'alphabétisation ne proposent pas de sessions à des niveaux dépassant les premiers stades de l'alphabétisation et de la post-alphabétisation, et les apprenants stagnent, se découragent finalement et abandonnent, ce qui peut aller jusqu'à un retour à l'illettrisme. Enfin, même les personnes faisant des efforts importants pour s'alphabétiser malgré les charges de travail qui leur incombent et ayant des projets pour servir leur communauté se voient bloquer dans leur progression pour des raisons apparemment politiques, mais en réalité à cause d'une mentalité de rivalités entre familles et entre individus qui perdure chez les détenteurs de pouvoir et les décideurs villageois et familiaux.

Face à toutes ces limites, l'enthousiasme des premiers temps a cédé la place depuis quelques années à un pulaar écrit qui se maintient grâce à des programmes d'alphabétisation nationaux ponctuels, coordonnés par l'État mais mis en place par des intervenants locaux (la politique du faire-faire) et financés par des bailleurs internationaux telle que la Banque mondiale. Les participants à ces programmes sont inscrits par les alphabétiseurs du village qui ont toute latitude pour désigner ceux qui pourront ou devront suivre les cours d'alphabétisation, car même les inscriptions ne sont plus toujours spontanées, et l'on dresse les listes des futurs apprenants en fonction de critères plus ou moins objectifs et en tous cas liés à des activités comme le maraîchage des femmes.

Parallèlement au désenchantement en direction de l'alphabétisation en pulaar, ces dernières années ont vu dans nos deux villages une reprise assez impressionnante du système formel qui pourtant avait largement montré ses limites au cours des décennies précédentes. À Dungel, l'ancienne école a été fermée et un nouvel établissement a été construit sur un autre site, sur financement de la Banque africaine de Développement, et le nombre de classes a augmenté progressivement. À Juude, le nombre de classes a quadruplé en trois ans sous l'impulsion notamment d'un nouveau directeur issu d'un village voisin qui a su impliquer l'ensemble des acteurs et des forces vives du village ainsi que les migrants et autres ONG ou bailleurs. Et les recrutements d'élèves ne posent pas de problème aux enseignants : il y aurait maintenant, à chaque rentrée scolaire, toujours beaucoup plus de candidats à inscrire que n'en peuvent accueillir les structures existantes et les équipes pédagogiques sous-payées et insuffisamment formées.

La demande est donc à nouveau forte, ou plutôt enfin forte pour la première fois, car les beaux jours de l'école publique aux lendemains des 
Indépendances correspondaient en réalité à des effectifs très réduits par rapport à l'échelle de la population d'enfants en âge d'être scolarisés. De plus, à cette époque, une certaine présélection des inscrits s'effectuait de fait, de diverses façons, en faveur des familles influentes de chaque village, alors qu'aujourd'hui, l'accès semble beaucoup plus ouvert à l'ensemble des composantes sociales de la communauté.

En somme, de cette petite histoire des grands moments des filières éducatives chargées d'assurer la pérennité des langues de l'écrit et des valeurs qu'elles véhiculent, on peut dégager les réflexions suivantes.

Tout d'abord, on voit combien chaque système est relativement fragile, ou en tout cas sujet à des fluctuations liées à la fois à des limites intrinsèques et à des interactions avec les autres filières de savoirs. Mais on a vu aussi que les liens de cause à effet entre la baisse d'un système et la croissance d'un autre ne sont pas si simples et systématiques et l'on ne peut en venir à considérer que la concurrence est le seul déterminant jouant sur les filières. Motivations et facteurs spirituels, culturels, économiques et conjoncturels interviennent très largement sur les «courbes de succès » ou dans les mutations de fond que connaissent les «écoles» au sens large.

Notons aussi qu'il ne semble pas y avoir de périodes creuses où les gens se détourneraient de toute éducation formelle ou informelle. Il y a longtemps eu une orientation prédominante vers l'une ou l'autre des filières éducatives, comme si elles se relayaient entre elles pour mieux perdurer, malgré leur rivalité latente et enfouie. Cela prouve au moins l'intégration dans les esprits de la nécessité d'une formation pour que les adultes d'aujourd'hui (alphabétisation en pulaar) ou de demain aient le maximum de chances de «devenir quelqu'un », au service des leurs bien entendu.

À l'heure actuelle, on a plutôt le sentiment qu'il y aurait un certain équilibre, nouveau, entre les filières, chacune présentant ses avantages et ses inconvénients mieux compris par les populations et recrutant des apprenants dans un corps social plus large (sexe et groupes statutaires concernant toutes les filières ; âge pour le pulaar et les enseignements des marabouts).

Sur le plan social, on assiste au fil du temps à une démocratisation de l'ensemble des filières, qui connaît cependant encore bien des limites liées, par exemple, aux disparités des ressources dont disposent les familles. Cette démocratisation de l'accès à une formation repose sur l'atténuation progressive des privilèges et exclusivités des castes nobles et sur celle des clivages caractérisant les relations entre groupes statutaires. On observe en effet que l'ancienne répartition selon le rattachement statutaire des enfants dans les filières s'est mutée en deux types de répartitions : l'une est intra-familiale, l'autre est une « répartition convergente » (ou absence de répartition). Outre l'attitude - rare, mais que l'on rencontre encore — de familles qui ne se préoccupent pas de dispenser à leurs enfants une éducation autre que l'éducation non formelle reçue dans leur environnement quotidien, les deux types de répartition évoqués correspondent à des stratégies opposées. 
La répartition intra-familiale consiste en ce qu'une fratrie soit répartie, sur choix prépondérant du père dans presque tous les cas, entre dudal et école publique, auxquels il faut adjoindre la « filière ménagère ou agricole » tant il est rarissime de voir des familles au sein desquelles la totalité des enfants ont suivi un parcours éducatif donné. On réduit ainsi les risques que telle ou telle filière ne soit pas un «bon placement» et on mise à l'inverse sur les chances de réussite du jeune, via des secteurs d'activités divers, et sur une représentativité la plus large possible de la famille dans le champ social, politique (au sens anthropologique) et économique. D'autres familles ont au contraire choisi délibérément d'envoyer tous leurs enfants à l'école publique ou auprès d'un marabout, considérant sans doute que telle filière est plus prometteuse que telle autre, selon des critères qui peuvent être spécifiques à chacune ou être communs aux deux. La logique est ici que tous les enfants ne sont pas aptes ou prédestinés par Dieu à réussir et qu'il vaut donc mieux concentrer ses ressources filiales sur la voie éducative que l'on estime la plus porteuse des espoirs que l'on entretient. Cette logique est renforcée par le fait qu'il faut de plus en plus compter avec l'initiative individuelle des enfants (abandon ou refus d'apprendre) qui aujourd'hui vient contrarier les projets des parents.

Dans un cas comme dans l'autre, nous sommes bel et bien dans le cadre d'une pensée communautariste, à l'échelle des familles, et le bien-être de l'enfant en tant qu'individu importe moins que l'intérêt général de l'entité familiale tel qu'il est perçu par le chef de famille. On est encore loin d'une conception de l'éducation qui se centrerait sur l'enfant pour lui donner le choix d'un enseignement et la possibilité de poursuivre là où il montre des aptitudes ou du plaisir, ce qui à terme serait sans doute aussi efficace en termes de réussite du jeune et donc non dénué d'un certain pragmatisme. Mais pour en arriver à cela — quand bien même ce serait souhaitable il faudrait que les différents types d'enseignement soient à même de rivaliser pour qu'il n'y ait pas péril de telle ou telle filière, et qu'ils mettent davantage au cœur de leurs approches une conception de l'enfant fondée sur plus d'estime et qui serait plus valorisante pour celui-ci. Mais une telle transformation impliquerait de revoir simultanément une bonne partie des fondements de la pensée et du fonctionnement des sociétés africaines.

\section{La promesse des lettrismes}

Après avoir montré la diversité des liens entre les langues/écritures ou entre les systèmes d'enseignement de celles-ci, au temps présent et au fil du temps, il nous paraît important de nous arrêter sur les valeurs symboliques ou concrètes dont est porteur chaque système écrit du point de vue des principaux concernés, afin de mieux cerner l'importance de ce qui se joue à travers les évolutions de chaque filière éducative. C'est ainsi que nous verrons finalement combien chaque sphère scripturo-linguistique est chargée 
d'une mission donnée qui à la fois compose et résume les représentations correspondant aux trois filières « savantes ».

L'enseignement de l'arabe coranique utilise le mode écrit, mais il faut bien comprendre que son but n'est pas tant l'accumulation de savoirs inscrits dans des livres que la transformation de l'être, son élévation vers Dieu et sa piété. L'écrit est un support pour stabiliser et mémoriser un contenu sacré qui doit pénétrer pleinement l'individu. D'ailleurs, il n'est pas rare de rencontrer en islam des maîtres coraniques aveugles, qui ont si bien intériorisé les versets coraniques et autres écrits liés aux sciences ou à la littérature islamiques que leur cécité ne représente pas un handicap majeur dans l'exercice de leurs fonctions (Santerre 1973 : 129).

On retiendra trois fonctions que remplit l'école coranique :

- La première est une fonction religieuse : tout musulman haalpulaar (croyant réellement en Dieu et adhérant à l'islam) est convaincu que pour pouvoir entrer au paradis après sa mort terrestre, il lui faut former ses enfants en les envoyant auprès d'un marabout afin qu'ils soient en mesure à leur tour de prier (les cinq prières quotidiennes) et d'être ainsi un musulman digne de ce nom qui s'achemine vers l'accomplissement des cinq piliers de l'islam.

— La deuxième fonction est identitaire et culturelle : les Haalpulaar'en s'attachent, tout autant qu'à assurer la pérennisation de leur langue, à pratiquer l'islam autant que possible et aux yeux de tous, tout comme l'ensemble des adeptes des confréries sénégalaises. Cela fait partie à la fois d'une identité que l'on se construit chaque jour, même si cela ne constitue pas en soi le but premier de la pratique religieuse, et d'une légitimisation de cette même identité culturelle. D'autant que l'islam fort majoritaire en milieu haalpulaar est un islam tidjane qui se doit de se perpétuer face à une «mouridisation » réelle ou projetée, parallèle à la wolofisation du pays.

- Enfin, bien qu'elle s'estompe progressivement, les dude ont une mission sociale à remplir : il s'agit de renouveler ou de créer des liens d'alliance ou de filiation spirituelle entre d'une part des individus et leur galle (maison ou lignage patriarcal) et d'autre part une famille maraboutique ou un marabout ayant une «valeur» sociale et spirituelle donnée. On reproduit ou on recrée ainsi un tissu de réseaux d'influence, de solidarité et de clientélisme sur lesquels s'appuyer dans certaines circonstances. Les enjeux politiques locaux et leurs fameuses «tendances» (groupes de partisans-clients opposés) se préoccupent d'ailleurs de rallier à leur cause les marabouts qui sont des personnalités incontournables de la vie du village, de ses méandres et de leurs dénouements. À l'inverse, comme ce fut le cas à Dungel à l'occasion d'une succession controversée à la fonction d'imam, les tendances politiques peuvent vampiriser le différend pour faire jouer pleinement le rapport 
de force et qu'une tendance soit reconnue plus forte que l'autre suivant l'issue du conflit.

Les enjeux liés à la fréquentation d'un duqal et au choix du marabout sont donc divers et importants pour les parents comme pour les futurs adultes. Concernant le choix du marabout à qui l'on confiera ses enfants, il faut ajouter que des critères beaucoup plus terre-à-terre viennent souvent le déterminer, comme, par exemple, la proximité du lieu d'habitation par rapport à celui de l'enfant, ou le fait qu'on le connaisse mieux, tout simplement, que d'autres marabouts du village, i.e. que l'on est déjà son «client ».

Si l'on peut retenir le paradis comme valeur phare de la filière coranique, celle du mariage symbolisera l'école publique. Il y a de cela quelques décennies, la terre représentait la source principale de revenus pour les ménages haalpulaar'en de la Vallée. On consommait presque exclusivement ce que l'on exploitait et si l'on avait des besoins autres qu'alimentaires, on vendait une partie de la récolte pour avoir du numéraire qui permette des achats (ou l'on troquait). Aujourd'hui, sous l'influence des modes alimentaires urbaines, entre autres ${ }^{25}$, on mange essentiellement du riz (tous les midis au moins), qui n'est pas cultivé par les villageois mais acheté, en provenance d'ailleurs plus souvent de Thaïlande ou des États-Unis que du Sénégal. Pour acheter ce riz, il faut soit vendre le mil produit localement et que l'on consommait auparavant en grande quantité, soit travailler dans des secteurs où l'on touche directement un salaire qui rend possible l'achat de riz. Et pour avoir des métiers permettant de gagner un minimum d'argent, il faut quitter le village et «tenter sa chance » ailleurs.

Or, les villageois ont à présent bien intégré l'atout que constitue l'éducation en français pour accéder à beaucoup de métiers urbains, et notamment pour décrocher des postes aussi rémunérateurs que possible, même s'il est également devenu clair dans les esprits que cette formation scolaire ne peut être une garantie, un sésame assurant automatiquement l'ascension sociale et la réussite matérielle. Les données de notre recensement confirment d'ailleurs parfaitement le fait qu'il y ait une corrélation marquée entre scolarisation et migration des hommes, notamment migration à l'étranger.

Mais quel est le rapport entre le mil, le riz, l'argent et le mariage ? C'est que tout simplement, les mariages reposent toujours sur une dot, et qui dit dot dit aujourd'hui argent, et non mesures de mil comme dans le passé. Pour épouser une jeune fille ou une femme représentant un bon parti, il devient donc plus important d'être scolarisé pour avoir davantage de chances de gagner un maximum d'argent plus tard que d'être issu de telle ou telle famille. Car même si le dernier aspect n'est pas négligé, loin s'en faut, il devient moins incontournable qu'il ne l'était autrefois. Les jeunes d'ailleurs se fréquentent maintenant dans les villages sans trop se

25. Ce passage du mil au riz tire avant tout son origine dans le contexte des grandes sécheresses des années 1970. 
cacher, et ce quelle que soit leur «caste», ignorant bien souvent — ou feignant d'ignorer — de quels groupes statutaires ils sont issus ou ce que cette appartenance statutaire impliquerait théoriquement aux yeux de leurs ascendants.

Ainsi, l'école «française » devient un atout pour la migration, et un moyen de partir pour mieux revenir et pouvoir mieux s'insérer dans le tissu social local via un mariage éventuellement plus valorisant au regard de la hiérarchie statutaire ou matérielle, ou correspondant davantage au souhait personnel du ou des futurs époux. Et il va sans dire qu'à l'heure actuelle, l'aspect « acculturation » ou appréhension de perdre son enfant, si ce dernier est scolarisé, a cédé la place aux impératifs de survie des villages qui dépendent très largement de la rente migratoire.

Quant à l'alphabétisation en pulaar, c'est la terre qui peut synthétiser l'ensemble des valeurs qui s'y rattachent. En effet, le pulaar est d'abord dans notre contexte la langue maternelle, et comme les concepts de langue et de terre sont associés au milieu d'origine du sujet, le pulaar est relié spontanément et intimement au terroir où les sujets ont grandi. Ensuite, les sessions d'alphabétisation touchent principalement les non-migrants, ceux qui restent en permanence au village ou ne le quittent que pour de courtes durées, alors que les études islamiques et les études scolaires amènent systématiquement le jeune, tôt ou tard, à quitter son village natal pour aller se former auprès de maîtres plus avancés dans d'autres villages ou dans des villes, où collèges et lycées pourront les accueillir. Enfin, et surtout, le pulaar est devenu un mode d'accès à l'exercice de certaines fonctions locales. Sans évoquer le cas des élus locaux qui rechignent à s'alphabétiser en pulaar, alors que l'exercice de leurs fonctions rendrait cette démarche plus que souhaitable, et qui, plus en amont dans la Vallée, demandent même à être payés par des ONG pour accepter de suivre les formations en langues nationales proposées ${ }^{26}$, la plupart des activités agricoles et de services promues par des ONG ou mises en place dans le cadre d'associations villageoises de développement ou autres groupements féminins maraîchers préconisent, voire imposent une maîtrise de l'écrit en pulaar. Même l'accès au crédit, avec le Programme intégré de Podor, se fait en pulaar. Et si cela est parfois contourné par le fait que les fonctions-clés des associations sont occupées par des analphabètes qui se font seconder par des fulanisants, il n'en reste pas moins que ces derniers ont un certain accès à des responsabilités importantes pour la vie de la communauté.

26. Selon les propos de Mamadou N'diaye qui intervient dans ce domaine dans le cadre des activités d'appui à la décentralisation par des formations en langues nationales mises en place par la SAFEFOD sur financements essentiellement américains. 
Pour conclure sur ces quelques aspects que nous avons esquissés des langues de l'écrit au Fuuta Tooro, nous retiendrons qu'au-delà des rapports de concurrence entre langues - concurrence avérée ou appréhendée et subjective - , on observe surtout à l'heure actuelle des relations de complémentarité et d'interactions à l'échelle de chaque village. Cette complémentarité s'exerce dans un contexte de tension entre préservation de l'ordre social établi et rupture avec celui-ci, en particulier ruptures entre volonté des parents et initiatives des enfants, entre principes de relations sociales dictés par les appartenances aux groupes statutaires et relations entre individus non régies par ces principes, entre répartition «traditionnelle » des tâches en fonction du sexe et autonomisation en douceur des femmes par rapport aux ressources du mari ou à ses souhaits initiaux.

Sur l'ampleur et l'ancrage du mouvement pulaar, on enregistre un certain fléchissement de l'enthousiasme sur le terrain, et un certain décalage entre l'image enjolivée de ce mouvement qu'ont pu donner les acteurs œuvrant dans le domaine de l'alphabétisation en pulaar et ses réalités dans une zone certes particulièrement reculée de la Vallée, qui ne connaît ni électricité, ni téléphone ni routes praticables.

À l'image des «flux et reflux du peuplement d'une rive à l'autre du fleuve Sénégal » (Schmitz 1999a : 2), on a l'impression d'assister en matière de langues et d'éducation à un ballottement des Haalpulaar'en selon une logique de réaction à des stimuli extérieurs, de rejet, d'adaptation, voire d'instrumentalisation, qui semblerait plus ou moins se stabiliser, mais dans quelle mesure et pour combien de temps ? Étant donné les contextes religieux, historique et culturel de la communauté haalpulaar, il semble en effet inconcevable qu'il en fut autrement, ce qui n'empêche pas de souhaiter une plus grande coopération et concertation entre les filières éducatives à l'avenir et une réflexion de fond à engager en partenariat avec les intéressés sur la place de l'enfant dans cette société.

Depuis le phénomène socioculturel qu'a constitué le mouvement pulaar, on se trouve donc toujours dans une diversité d'offres linguistico-éducatives qui permet à chaque langue écrite ou à chaque système de savoirs de se maintenir en place et de répondre ainsi à des demandes qui se juxtaposent très souvent chez les individus comme parmi les entités sociales. Malheureusement, ces trois filières réunies ne parviennent pas à enrôler l'ensemble des individus d'une tranche d'âge, loin s'en faut, et la majorité des villageois est encore et malgré tout analphabète ou illettrée, ce que la plupart d'entre eux regrettent et qui constitue à leurs propres yeux un handicap majeur qui se répercute sur leur vie quotidienne personnelle comme sur celle de l'ensemble de la communauté. De nouvelles voies restent donc sans doute à imaginer et de nombreux efforts à développer pour que chacun trouve une place sur l'ensemble de la palette linguistico-scripturale et éducative des villages et que cela se fasse en harmonie avec les aspirations des individus et pour le bien de la collectivité.

EHESS-ENS-IRD, Paris. 


\section{BIBLIOGRAPHIE}

ARED

2000 Rapport d'évaluation du programme d'alphabétisation dans la zone des quatre forages du Ferlo, Dakar, ARED.

Botте, R.

1990 «Pouvoir du Livre, pouvoir des hommes : la religion comme critère de distinction », Journal des Africanistes, LX (2) : 37-53.

Diop, B., Faye, A., Sylla, Y. \& Gueye, A.

1990 L'impact des journaux en langues nationales sur les populations sénégalaises, Dakar, Association des chercheurs sénégalais.

ERNY, P.

1972 L'enfant et son milieu en Afrique noire. Essais sur l'éducation traditionnelle, Paris, Payot.

FAGERBERG-DiALlo, S.

1995 «Milk and Honey: Developing Written Literature in Pulaar», Yearbook of Comparative and General Literature, $43: 67-83$.

1996 L'éducation et la lecture en langue pulaar: le cas du Sénégal, Dakar, ARED-GIPLLN.

1997 «Création d'un milieu lettré en langues nationales (l'exemple du pulaar au Sénégal) », Développement et Coopération, 1: 16-18.

HUMERY, M.-È.

1997 Facteurs et enjeux du développement écrit d'une langue africaine: le cas du mouvement pulaar au Sénégal (1960-1996), Thèse de $3^{\text {e }}$ cycle, Paris, Université Paris I.

KAnE, M.

1997 «Du pâtre au patron de la "Révolution Al-Fatah": la vie et l'œuvre d'El Hadjj Mahmoud Bâ Jowoli (1905-1978) », in D. RoBinson \& J.-L. Triaud, Les temps des marabouts, itinéraires et stratégies islamiques en Afrique occidentale française v. 1880-1960, Paris, Éditions Karthala.

LÉON, A.

1991 Colonisation, enseignement et éducation, Paris, L’Harmattan.

MBACKe, K.

1994 «Daaras et droits de l'enfant», Études islamiques, 3.

M'ВокоLо, E.

1992 Afrique Noire. Histoire et civilisations, tome II, XIX ${ }^{e}-X X^{e}$ siècles, Paris, Hatier-AUPELF. 
Mohamadou, A.

2000 «Nouvelles tendances en littérature peule. Présentation des textes de cinq auteurs Haal-pulaar (Sénégal et Mauritanie)», in U. BAUMGardT \& A. Bounfour, eds, Panorama des Littératures africaines. État des lieux et perspectives, Paris, L'Harmattan : 77-92.

SALVAING, B.

s. d. «Langues nationales et développement d'un environnement lettré en Sénégambie », non publié, Centre de recherches africaines.

SANTERRE, R.

1973 Pédagogie musulmane d'Afrique noire, l'école coranique peule du Cameroun, Montréal, Presses de l'Université de Montréal.

Schmitz, J.

1986 «L'État géomètre : les leydi des Peul du Fuuta Tooro (Sénégal) et du Maasina (Mali)», Cahiers d'Études africaines, XXVI (3), 103 : 349-394.

1993 Lexique des catégories sociales, non publié.

1994a «Cités noires : les républiques villageoises du Fuuta Tooro (Vallée du fleuve Sénégal) », Cahiers d'Études africaines, XXXIV (1-3), 133-135 : 419-460.

1994b «Peuls : Histoire, système religieux et rituels », non publié.

1998 «Introduction», in S. M. KAMARA \& J. Schmitz, eds, Zuhur al Basatin. L’histoire des musulmans noirs de la Vallée du Sénégal, vol. 1, La révolution des Toorobbe musulmans, Paris, Éditions du CNRS : 9-91.

1999a «L'expulsion des Fulbe de la rive mauritanienne du fleuve Sénégal en 1989 : répétition dans l'histoire ou catastrophe », in V. AzANYA \& A. BREEDVELD et al., Pastoralists under Pressure?, Leiden-Boston-Köln, Brill : 329-369.

$1999 \mathrm{~b}$ «Joutes de langue et figures de style», in R. Botte, J. Boutrais \& J. Schmitz, eds, Figures peules, Paris, Éditions Karthala : 19-31.

2000 «Le souffle de la parenté. Mariage et transmission de la baraka chez les clercs musulmans de la Vallée du Sénégal », L’Homme, 154 : 241-278.

\section{RÉSUMÉ}

Dans la Vallée du fleuve Sénégal (Sénégal et Mauritanie), le pulaar (ou peul) a connu un phénomène socioculturel important depuis deux décennies. Cette langue s'est en effet scripturalisée grâce à des activités d'alphabétisation qui se sont développées spontanément ou en lien avec des ONG, dans tous les villages haalpulaar'en. À travers l'étude sociologique de deux villages, on s'intéresse ici non seulement à l'écriture de cette langue, mais aussi à celle de l'arabe coranique et du français. Les filières de formation ou d'éducation dans chacune de ces langues (cours d'alphabétisation, " école » coranique et école publique) seront considérées comme des révélateurs des liens et interactions entre ces trois langues de l'écrit. L'histoire de ces langues écrites et les valeurs qui s'y rattachent permettront de cerner les enjeux liés au mode écrit et à l'éducation dans une zone très enclavée où les moyens de communication modernes sont inexistants (routes, téléphone, etc.). 


\section{ABSTRACT}

Paradise, Marriage and Earth: Literacy, Writings and Languages in Fuutanke Area (Arabic, french and fula). - In the Valley of the Senegal River (Mauritania and Senegal), fula has known an important socio-cultural phenomena during the last decades. This language has been developped in his writing form by literacy trainings that took place in every haalpulaar'en villages, spontaneously or with the support of NGOs. By the sociological approach of two villages, we deal not only with the issues of writing in this mother language, but also with those concerning koranic arabic and french. Literacy trainings, koranic schools and public schools are considerated as revealing the links and interactions between these three scripted languages. The history of these languages and the values relative to each one underline the importance of each of them and of the writing way of communication in an area where any modern technologies or infrastructures of communication can't be found.

Mots-clés/keywords : Coran, écriture, éducation, langue peule, plurilinguisme/Koran, writing culture, education, literacy, fula. 